Article

\title{
Production of New Isoflavone Diglucosides from Glycosylation of 8-Hydroxydaidzein by Deinococcus geothermalis Amylosucrase
}

\author{
Chien-Min Chiang ${ }^{1,+}{ }^{+}$, Tzi-Yuan Wang ${ }^{2,+}+\mathbb{D}$, Jiumn-Yih Wu ${ }^{3,+}$, Yun-Rong Zhang ${ }^{4}$, Shu-Yuan Lin 4 \\ and Te-Sheng Chang $4, * \mathbb{D}$
}

Citation: Chiang, C.-M.; Wang, T.-Y.; Wu, J.-Y.; Zhang, Y.-R.; Lin, S.-Y.; Chang, T.-S. Production of New Isoflavone Diglucosides from Glycosylation of 8-Hydroxydaidzein by Deinococcus geothermalis Amylosucrase. Fermentation 2021, 7 , 232. https://doi.org/10.3390/ fermentation7040232

Academic Editors:

Clemencia Chaves-López and Alexander Rapoport

Received: 23 August 2021

Accepted: 14 October 2021

Published: 16 October 2021

Publisher's Note: MDPI stays neutral with regard to jurisdictional claims in published maps and institutional affiliations.

Copyright: (c) 2021 by the authors. Licensee MDPI, Basel, Switzerland. This article is an open access article distributed under the terms and conditions of the Creative Commons Attribution (CC BY) license (https:// creativecommons.org/licenses/by/ $4.0 /)$.
1 Department of Pharmacy, Chia Nan University of Pharmacy and Science, No. 60, Sec. 1, Erh-Jen Rd., Jen-Te District, Tainan 71710, Taiwan; cmchiang@mail.cnu.edu.tw

2 Biodiversity Research Center, Academia Sinica, Taipei 11529, Taiwan; tziyuan@gmail.com

3 Department of Food Science, National Quemoy University, Kinmen County 892, Taiwan; wujy@nqu.edu.tw

4 Department of Biological Sciences and Technology, National University of Tainan, Tainan 70005, Taiwan; S10758011@gm2.nutn.edu.tw (Y.-R.Z.); doreen890513@gmail.com (S.-Y.L.)

* Correspondence: mozyme2001@gmail.com; Tel./Fax: +886-6-260-2137

+ These authors contributed equally to this manuscript.
Abstract: 8-Hydroxydaidzein (8-OHDe) is a non-natural isoflavone polyphenol isolated from fermented soybean foods. 8-OHDe exhibits a wide range of pharmaceutical activities. However, both the poor solubility and instability of 8-OHDe limit its applications. To resolve the limitations of 8-OHDe, Deinococcus geothermalis amylosucrase (DgAS) has previously been used to glycosylate 8-OHDe to produce soluble and stable 8-OHDe-7-O- $\alpha$-glucopyranoside (8-OHDe-7-G) in a $0.5 \mathrm{~h}$ reaction time. In this study, we aimed to use DgAS and an extended reaction time to produce 8-OHDe diglucosides. At least three 8-OHDe derivatives were produced after a $24 \mathrm{~h}$ reaction time, and two major products were successfully purified and identified as new compounds: 8 -OHDe-7-O- $[\alpha-$ glucopyranosyl-(1 $\rightarrow 6)$ - $\alpha$-glucopyranoside] (8-OHDe-7-G2) and 8-OHDe-7,4'-O- $\alpha$-diglucopyranoside (8-OHDe-7-G-4'-G). 8-OHDe-7-G-4'-G showed a 4619-fold greater aqueous solubility than 8-OHDe. In addition, over $92 \%$ of the 8 -OHDe diglucosides were stable after $96 \mathrm{~h}$, while only $10 \%$ of the 8-OHDe could be detected after being subjected to the same conditions. The two stable 8-OHDe diglucoside derivatives have the potential for pharmacological usage in the future.

Keywords: amylosucrase; Deinococcus geothermalis; 8-hydroxydaidzein; isoflavone; glycosylation

\section{Introduction}

8-Hydroxydaidzein (7,8,4'-trihydroxyisoflavone; 8-OHDe) is an artificial isoflavone derived from daidzein ( $7,4^{\prime}$-dihydroxyisoflavone). It can be isolated from fermented soybean sauces, such as Indonesian tempeh, Chinese douchi, Korean doenjang, and Japanese soybean koji and miso. During fermentation, unique microbial cytochrome P450 enzymes hydroxylate the soybean isoflavone daidzein to produce 8-OHDe [1].

Past studies have shown that 8-OHDe possesses a range of bioactivities, including: anti-cancer, anti-tyrosinase, skin whitening, anti-aldose reductase, and suppression of multidrug resistance activity (reviewed in [1]); anti-inflammatory activity [2,3]; suppression of ultraviolet B (UVB)-induced matrix metalloproteinase (MMP)-1 expression [4]; and neuroprotection [5] and memory-enhancing activity [6]. Based on the association of these bioactivities of 8-OHDe with pharmaceuticals and/or cosmeceuticals, 8-OHDe biotransformation processes were developed by genetically engineering Pichia pastoris and Escherichia coli (reviewed in [1]). In addition, Seo et al. (2013) and Wu et al. (2015) developed mass production processes to generate 8-OHDe from daidzein in Aspergillus oryzae [7,8]. Increases in the quality and quantity of 8-OHDe provide more opportunities for industrial applications. 
However, certain drawbacks (e.g., low aqueous solubility and instability) limit the use of isoflavones in pharmaceuticals and cosmeceuticals [9]. One solution involves chemically or enzymatically glycosylating these molecules to improve their solubility and stability [10]. A comparison of these two methods showed that enzymatic glycosylation of flavonoids using glycosyltransferases (GTs) and glycoside hydrolases (GHs) offers more advantages than chemical methods [11]. GTs glycosylate flavonoids via a $\beta$-glycosidic linkage. In contrast, GHs glycosylate flavonoids via an $\alpha$-glycosidic linkage. Both $\alpha$-glycosidic and $\beta$-glycosidic flavonoids are more soluble than flavonoids [12,13]. However, GHs use cheaper sugars, such as starch, maltodextrin, maltose, and sucrose, as donors during glycosylation [14], whereas GTs use expensive uridine diphosphate-glucose (UDP-G) as the sugar donor. Therefore, GHs are generally preferred for the bioindustrial production of glycosylated molecules.

Amylosucrase (AS, E.C. 2.4.1.4) is a versatile sucrose-hydrolyzing enzyme that belongs to GH family 13 (GH13) [15]. AS can catalyze the synthesis of $\alpha$-1,4-glucans using sucrose as the sole substrate. Furthermore, it can mediate the glycosylation of various small molecules with sucrose. Thus, recombinant Deinococcus geothermalis amylosucrase (DgAS) and sucrose were used to glycosylate 8 -OHDe to generate 8-OHDe-7-O- $\alpha$-glucopyranoside (8-OHDe-7-G) in a reaction time of $0.5 \mathrm{~h} \mathrm{[13].} \mathrm{Recently,} \mathrm{Rha} \mathrm{et} \mathrm{al.} \mathrm{(2019)} \mathrm{reported} \mathrm{that} \mathrm{DgAS}$ could glycosylate the daidzein glucoside daidzin (daidzein-7-O- $\beta$-glucoside) to form a

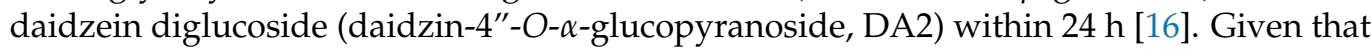
the chemical structure of 8-OHDe-7-G is similar to that of daidzin, we theorized that DgAS might produce other 8 -OHDe glycosides in a $24 \mathrm{~h}$ reaction time and investigated this in the current study. We did indeed find two novel diglucoside derivatives of 8-OHDe.

\section{Materials and Methods}

\subsection{Enzymes and Chemicals}

8-OHDe was purified following the biotransformation of daidzein by A. oryzae according to the method reported by Wu et al. [8]. Recombinant DgAS was produced by a recombinant $E$. coli (DE3) harboring the pETDuet-DgAS expression vector according to the method reported previously [13]. Soluble DgAS was then successfully purified using $\mathrm{Ni}^{2+}$ chelate affinity chromatography, and the purity was confirmed using sodium dodecyl sulfate polyacrylamide gel electrophoresis (SDS-PAGE) (Figure S1). The specific sucrose hydrolysis activity of DgAS was determined to be $6.6 \mathrm{U} / \mathrm{mg}$ using a 3,5-dinitrosalicylic acid (DNS) solution and a previously described method [16]. Briefly, a reaction mixture containing $25 \mu \mathrm{g} / \mathrm{mL}$ DgAS with $25 \%(w / v)$ sucrose in $50 \mathrm{mM}$ of phosphate buffer (PB) at $\mathrm{pH} 7$ was incubated at $40{ }^{\circ} \mathrm{C}$ for $10 \mathrm{~min}$. After the reaction was stopped by boiling, the resultant reducing sugars were estimated using the DNS method. One unit of DgAS activity was defined as the amount of DgAS that hydrolyzed sucrose into $1 \mu \mathrm{mol}$ of fructose per min.

\subsection{Biotransformation}

The reaction mixture $(1 \mathrm{~mL})$ comprised $25 \mu \mathrm{g} / \mathrm{mL}$ DgAS, $1 \mathrm{mg} / \mathrm{mL} \mathrm{8-OHDe,} \mathrm{10 \%}$ $(w / v)$ sucrose, and $50 \mathrm{mM} \mathrm{PB}$ at $\mathrm{pH} 7$ and was incubated at $40{ }^{\circ} \mathrm{C}$ for $24 \mathrm{~h}$, based on the previous study [13]. The reaction was stopped by adding an equal volume of methanol and analyzed using high-performance liquid chromatography (HPLC).

Conversion was calculated using the following formula and expressed as a percentage: (1-HPLC peak area of the residues of 8-OHDe divided by HPLC peak area of the initial 8 -OHDe) $\times 100$. The yield of each product was calculated using the following formula and expressed as a percentage: (HPLC peak area divided by HPLC peak area of the initial 8 -OHDe) $\times 100$.

\subsection{HPLC Analysis}

A combo system was used that consisted of an Agilent ${ }^{\circledR} 1100$ series HPLC system (Santa Clara, CA, USA) equipped with a gradient pump (Waters 600, Waters, Milford, 
MA, USA). The HPLC system was controlled via a PC workstation using Chromatography Data Station software (SISC, Scientific Information Service Co., LTD., Taipei, Taiwan). The stationary phase was a C18 column (Sharpsil H-C18, $5 \mu \mathrm{m}, 4.6$ i.d. $\times 250 \mathrm{~mm}$, Sharpsil, Beijing, China), and the mobile phase was $1 \%$ acetic acid in water (A) and methanol (B). The elution condition was as follows: a linear gradient from 0 min with $40 \%$ B to $20 \mathrm{~min}$ with $70 \% \mathrm{~B}$; isocratic elution from $20 \mathrm{~min}$ to $25 \mathrm{~min}$ with $70 \% \mathrm{~B}$; a linear gradient from 25 min with $70 \%$ B to $28 \mathrm{~min}$ with $40 \% \mathrm{~B}$; and isocratic elution from $28 \mathrm{~min}$ to $35 \mathrm{~min}$ with $40 \%$ B. The flow rate was $1 \mathrm{~mL} / \mathrm{min}$, the sample volume was $10 \mu \mathrm{L}$, and the detection condition was set at $254 \mathrm{~nm}$.

\subsection{Purification and Identification of the Biotransformation Metabolites}

To purify the biotransformation metabolites, the biotransformation reaction described above was scaled up to $30 \mathrm{~mL}$. After the reaction, purification was conducted using previously described methods [13]. Briefly, the mixture was filtered through a $0.2 \mu$ m nylon membrane, and the filtrate was injected into a preparative YoungLin HPLC system (YL9100, YL Instrument, Gyeonggi-do, Korea) equipped with a preparative C18 reversed-phase column (Inertsil, $10 \mu \mathrm{m}, 20.0$ i.d. $\times 250 \mathrm{~mm}$, ODS 3, GL Sciences, Eindhoven, Netherlands) to separate the biotransformation products. The operational conditions for the preparative HPLC were the same as those used for the analytical HPLC. The fractions corresponding to the metabolite peaks identified during the HPLC analysis were collected, condensed under a vacuum, and then lyophilized. Finally, 3.3, 12.8, and $6.7 \mathrm{mg}$ of compounds (1), (2), and (3), respectively, were obtained from the $30 \mathrm{~mL}$ reaction, and the compound structures were confirmed with mass spectral analysis and nuclear magnetic resonance (NMR). The mass spectral analysis was performed using a Finnigan LCQ Duo mass spectrometer (ThermoQuest Corp., San Jose, CA, USA) with electrospray ionization (ESI). ${ }^{1} \mathrm{H}-$ and ${ }^{13} \mathrm{C}-$ NMR, distortionless enhancement by polarization transfer (DEPT), heteronuclear single quantum coherence (HSQC), heteronuclear multiple bond connectivity (HMBC), correlation spectroscopy (COSY), and nuclear Overhauser effect spectroscopy (NOESY) spectra were recorded using a Bruker AV-700 NMR spectrometer (Bruker Corp., Billerica, MA, USA) at ambient temperature. Standard pulse sequences and parameters were used for the NMR experiments, and all chemical shifts were reported in parts per million (ppm, $\delta$ ).

\subsection{Determination of Solubility and Stability}

Aqueous solubility and stability were determined using previously described methods [13]. For the aqueous solubility assay, the tested compound was vortexed in deionized $\mathrm{H}_{2} \mathrm{O}$ for $1 \mathrm{~h}$ at $25^{\circ} \mathrm{C}$. The mixture was analyzed using HPLC. For the stability assay, the test compound stock solution ( $100 \mathrm{mg} / \mathrm{mL}$ in dimethyl sulfoxide) was diluted 100 -fold to $1 \mathrm{mg} / \mathrm{mL}$ in $50 \mathrm{mM}$ Tris buffer at $\mathrm{pH}$ 8.0. Then, aliquots were taken for HPLC analysis at the determined time intervals.

\section{Results and Discussion}

\subsection{Biotransformation of 8-OHDe by DgAS over $24 \mathrm{~h}$}

Rha et al., (2019) indicated that DgAS can glycosylate an isoflavone glucoside (daidzin) to form daidzin glucosides after a $24 \mathrm{~h}$ reaction time [16]. Our previous study also revealed that DgAS can glycosylate 8-OHDe to form an isoflavone glucoside (8-OHDe-7-G) within $0.5 \mathrm{~h}$. Therefore, this study was designed to determine whether 8-OHDe-7-G could be glycosylated by DgAS over a $24 \mathrm{~h}$ period. The reaction mixtures were analyzed using HPLC. Figures 1 and 2 show the HPLC analysis and time course for the 8-OHDe derivatives formed by DgAS after various reaction times. The results demonstrate that 8-OHDe was glycosylated by DgAS to form 8-OHDe-7-G after $0.5 \mathrm{~h}$. The production of 8-OHDe-7-G was at its maximum after $1 \mathrm{~h}$ and then decreased gradually (Figure 2). The other three products, compounds (1) to (3), also appeared after $1 \mathrm{~h}$ (Figure 2). Throughout the time course experiment, the amount of compound (1) was low and remained steady and stable. However, the large amounts of compounds (2) and (3) gradually increased from $1 \mathrm{~h}$ 
onwards. These results reveal that 8-OHDe-7-G can be glycosylated further by DgAS, which is consistent with the activity of DgAS on daidzin [16]. In contrast, no 8-OHDe glucoside was produced when heat-inactivated DgAS was used in the control reaction (Figure S2).

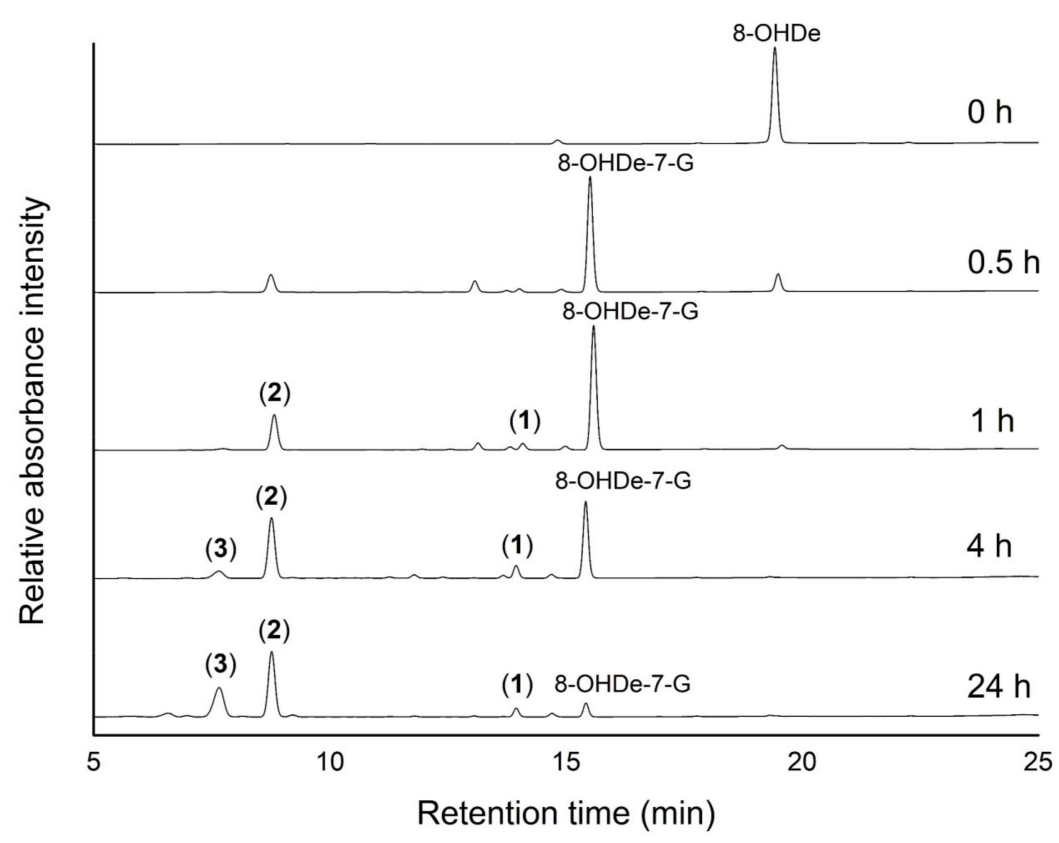

Figure 1. High-performance liquid chromatography (HPLC) analysis of the biotransformation products of $8-\mathrm{OHDe}$ produced by DgAS. The reaction was conducted with $10 \%(w / v)$ sucrose, $25 \mu \mathrm{g} / \mathrm{mL}$ DgAS, and $1 \mathrm{mg} / \mathrm{mL}$ 8-OHDe in $50 \mathrm{mM} \mathrm{PB}(\mathrm{pH}=7)$ at $40{ }^{\circ} \mathrm{C}$. At each time interval, the reaction mixture was analyzed using HPLC. The HPLC conditions are described in the Materials and Methods section.

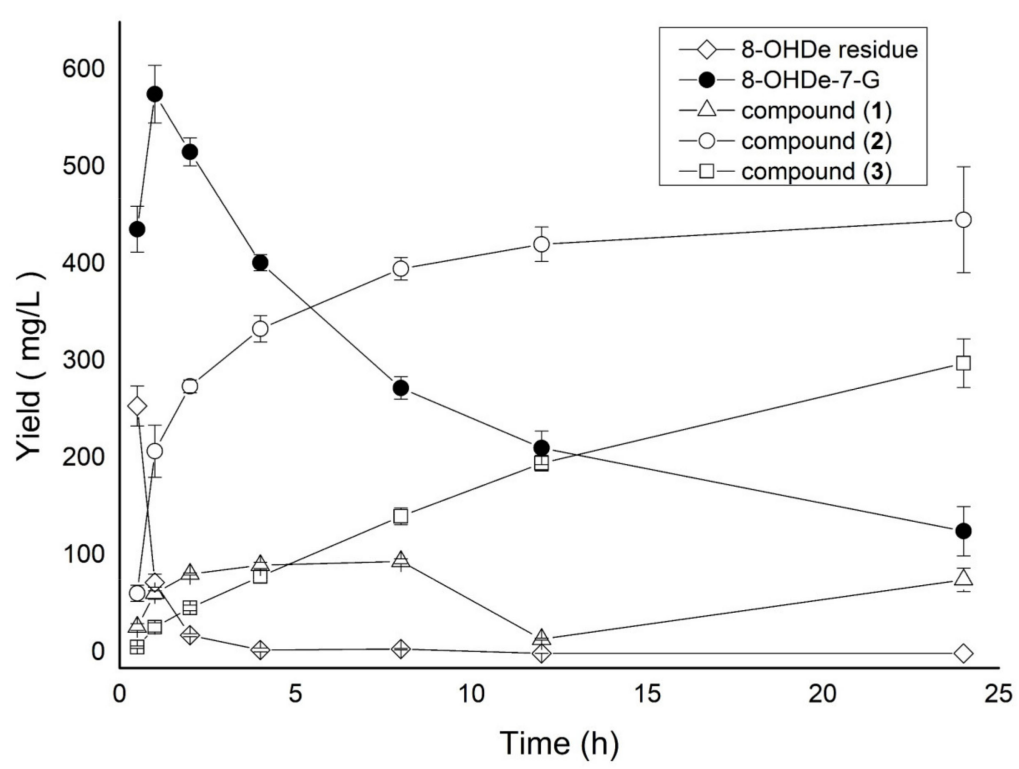

Figure 2. Time course of the production of 8-OHDe glucosides from the biotransformation of 8-OHDe by DgAS. The yields of the 8-OHDe glucosides were calculated using the HPLC curves shown in Figure 1. The methods used to calculate the residual 8-OHDe (open diamond) and to estimate the yields of 8-OHDe-G-7 (closed circle), compound (1) (open triangle), compound (2) (open circle), and compound (3) (open square) are described in the Materials and Methods section. 


\subsection{Purification and Identification of the Biotransformation Products}

To resolve the chemical structures of the new derivatives, the biotransformation was scaled up, and compounds (1), (2), and (3) were purified and resolved by mass spectral analysis. The mass spectral analysis of compounds (1) and (2) showed identical [M-H]- ion peaks at $\mathrm{m} / \mathrm{z} 593.2$ in the electrospray ionization mass (ESI-MS) spectrum corresponding to the molecular formula $\mathrm{C}_{27} \mathrm{H}_{30} \mathrm{O}_{15}$, which implied that compounds (1) and (2) were 8OHDe diglucosides [8-OHDe+2Glucose-2OH]- (Figures S3 and S4). In addition, compound (3) showed an ion peak at $\mathrm{m} / \mathrm{z} 755.2$ in the ESI-MS spectrum, which implied that it was 8OHDe triglucoside [8-OHDe+3Glucose-3OH]- (Figure S5). The structures of compounds (1) and (2) were further resolved by NMR spectral analysis, including ${ }^{1} \mathrm{H}$ and ${ }^{13} \mathrm{C}$ NMR, DEPT, COSY, NOESY, HSQC, and HMBC spectra. The ${ }^{1} \mathrm{H}$ - and ${ }^{13} \mathrm{C}-\mathrm{NMR}$ signal assignments were conducted accordingly (Table 1 and Figures S6-S20). In addition to the 8-OHDe signals, those related to each new compound revealed 14 proton signals (from 3.19 to $5.46 \mathrm{ppm}$ ) and 12 carbon signals (from 60.8 to $100.8 \mathrm{ppm}$ ), indicative of two glucopyranosyl moieties.

For compound (1), a downfield shift of the ${ }^{1} \mathrm{H}$ signal of $\mathrm{H} 6$ was observed compared to that of 8-OHDe. The $J$ coupling constants $(3.5 \mathrm{~Hz})$ for two anomeric protons $(5.32 \mathrm{ppm}$ and $4.65 \mathrm{ppm}$ ) in the ${ }^{1} \mathrm{H}-\mathrm{NMR}$ spectrum indicated the $\alpha$-configuration of the two glucose moieties. The cross-peak of H-" with C-7 (5.32/148.8 ppm) in the HMBC spectrum (Figure S12) and the cross-peak of H-1" with H-6 (5.32/7.33 ppm) in the NOESY spectrum (Figure S10) indicated that the first glucose moiety was connected to the C-7 hydroxyl group of 8-OHDe. The cross-peaks of H-1"' with C-6" (4.65/66.1 ppm) and H-6" with C-1"' (3.59/98.2 ppm and $3.73 / 98.2 \mathrm{ppm}$ ) in the HMBC spectrum indicated that the second glucose moiety was connected to the C-6" hydroxyl group of the first glucose.

For compound (2), significant downfield shifts of the ${ }^{1} \mathrm{H}$ signals of $\mathrm{H} 6, \mathrm{H} 2{ }^{\prime}\left(4^{\prime}\right)$, and $\mathrm{H}^{\prime}\left(5^{\prime}\right)$ were observed compared to those of 8-OHDe. The J coupling constants $(3.5 \mathrm{~Hz})$ for two anomeric protons $(5.46 \mathrm{ppm}$ and $5.42 \mathrm{ppm})$ in the ${ }^{1} \mathrm{H}-\mathrm{NMR}$ spectrum indicated the $\alpha$-configuration of the two glucose moieties. The cross-peaks of $\mathrm{H}-1$ " with C-7 (5.46/148.8 ppm) and H-1"' with C-4' (5.42/157.1 ppm) in the HMBC spectra (Figure S19), as well as the cross-peaks of H-1" with H-6 (5.46/7.36 ppm) and H-1"' with H-3'(5') (5.42/7.14 ppm) in the NOESY spectra (Figure S17), indicated that the two glucose moieties were individually connected to the hydroxyl group at C-7 or C-4' (the key HMBC and NOESY correlations of both compounds are shown in Figure S20).

From the mass spectral analysis and NMR data, compounds (1) and (2) were identified as 8-OHDe-7-O-[ $\alpha$-glucopyranosyl-( $1 \rightarrow 6)-\alpha$-glucopyranoside] (8-OHDe-7-G2) and 8-OHDe-7,4'-O- $\alpha$-diglucopyranoside (8-OHDe-7-G-4'-G), respectively. However, the NMR data for compound (3) showed multiple extra carbon signals, which indicated that compound (3) was an 8-OHDe triglucoside mixture. The exact glucoside linkages in this 8 -OHDe triglucoside mixture were not elucidated. Nevertheless, the chemical structures of both 8-OHDe-7-G2 (1) and 8-OHDe-7-G-4'-G (2) were confirmed. Figure 3 summarizes the biotransformation of 8-OHDe mediated by DgAS. During the catalytic process, it seems that DgAS preferentially glycosylates the C-7 hydroxyl group first and then the $4^{\prime}$-hydroxyl group of the 8-OHDe skeleton.

Rha et al., (2019) reported that DgAS glycosylates daidzin (daidzein-7-O- $\beta$-glucoside) to produce a daidzein diglucoside (daidzin- 4 "'-O- $\alpha$-glucopyranoside, DA2), and that the linkage between the two glucosyl moieties is $\alpha-(1 \rightarrow 4)$ [16]. In another study, Rha et al. (2020) reported that DgAS glycosylates isoquercitrin (quercetin-3-O-glucoside) to produce three quercetin glucosides (IQ-G1', IQ-G2', and IQ-G3), and that the linkages between the two glucosyl moieties are either $\alpha-(1 \rightarrow 2), \alpha-(1 \rightarrow 4)$, or $\alpha-(1 \rightarrow 6)$ [17]. This study further found that DgAS glycosylates 8-OHDe-7-G to generate a daidzein diglucoside (8-OHDe-7-G2), and that the linkage between the two glucosyl moieties is $\alpha-(1 \rightarrow 6)$ (Figure 3). These results revealed that DgAS has broad regioselectivity with regard to the formation of glycosidic linkages. In fact, amylosucrases naturally exhibit three catalytic functions (polymerization, isomerization, and hydrolysis [18]) and could create different glycosidic linkages, such as $\alpha-(1 \rightarrow 1), \alpha-(1 \rightarrow 3)$, or $\alpha-(1 \rightarrow 4)$, via polymerization and/or isomerization. Therefore, the 
broad regioselectivity of DgAS in terms of the glycosidic linkages of flavonoids could be expected and is consistent with the catalytic functions of amylosucrases.

Table 1. NMR spectroscopic data for compounds (1) and (2) (in DMSO- $d_{6} ; 700 \mathrm{MHz}$ ).

\begin{tabular}{|c|c|c|c|c|c|c|}
\hline \multirow{2}{*}{$\begin{array}{l}\text { Compound } \\
\text { Position }\end{array}$} & \multicolumn{3}{|c|}{$\begin{array}{c}\text { Compound (1) } \\
\text { 8-OHDe-7-O-[ } \alpha \text {-Glucopyranosyl-(1 } \rightarrow 6)-\alpha- \\
\text { Glucopyranoside] }\end{array}$} & \multicolumn{3}{|c|}{$\begin{array}{c}\text { Compound (2) } \\
\text { 8-OHDe-7, } 4^{\prime}-\mathrm{O}-\alpha \text {-Diglucopyranoside }\end{array}$} \\
\hline & $\delta_{\mathrm{C}}$ & $\delta_{\mathrm{H}}(J$ in $\mathrm{Hz})$ & НMBC & $\delta_{\mathrm{C}}$ & $\delta_{\mathrm{H}}(J$ in $\mathrm{Hz})$ & НМВС \\
\hline \multicolumn{7}{|l|}{$\begin{array}{l}\text { Isoflavone } \\
\text { moiety }\end{array}$} \\
\hline 2 & 153.1 & $8.39, \mathrm{~s}$ & & 154.0 & $8.43, \mathrm{~s}$ & \\
\hline 3 & 123.0 & & $\mathrm{H}-2, \mathrm{H}-2^{\prime}\left(6^{\prime}\right)$ & 125.8 & & $\mathrm{H}-2, \mathrm{H}-3^{\prime}\left(5^{\prime}\right)$ \\
\hline 4 & 175.4 & & $\mathrm{H}-2, \mathrm{H}-5$ & 175.6 & & $\mathrm{H}-2, \mathrm{H}-5$ \\
\hline 5 & 113.2 & 7.46, d (8.7) & & 114.1 & $7.47, \mathrm{~d}(8.9)$ & \\
\hline 6 & 115.3 & $7.33, \mathrm{~d}(8.8)$ & H-5 & 114.7 & $7.36, \mathrm{~d}(8.9)$ & $\mathrm{H}-5$, \\
\hline 7 & 148.8 & & $\begin{array}{l}\text { H-5, H-6, } \\
\text { Glc1-H-1" }\end{array}$ & 148.8 & & H-5, H-6, Glc1-H-1" \\
\hline 8 & 138.2 & & H-5 & 137.2 & & H-5, H-6 \\
\hline $8 \mathrm{a}$ & 146.3 & & $\mathrm{H}-2, \mathrm{H}-5$ & 146.4 & & $\mathrm{H}-2, \mathrm{H}-5, \mathrm{H}-6$ \\
\hline $4 a$ & 120.3 & & H-6 & 120.0 & & H-6, \\
\hline $1^{\prime}$ & 122.6 & & $\mathrm{H}-2, \mathrm{H}-3^{\prime}\left(5^{\prime}\right)$ & 123.0 & & $\mathrm{H}-2, \mathrm{H}-2^{\prime}\left(6^{\prime}\right), \mathrm{H}-3^{\prime}\left(5^{\prime}\right)$ \\
\hline $2^{\prime}\left(6^{\prime}\right)$ & 130.1 & $7.40, \mathrm{~m}$ & $\mathrm{H}-2^{\prime}\left(6^{\prime}\right), \mathrm{H}-3^{\prime}\left(5^{\prime}\right)$ & 130.3 & $7.50, \mathrm{~d}(8.6)$ & H-2' $\left(6^{\prime}\right), H-3^{\prime}\left(5^{\prime}\right)$ \\
\hline $3^{\prime}\left(5^{\prime}\right)$ & 114.9 & $6.81, \mathrm{~m}$ & $\mathrm{H}-2^{\prime}\left(6^{\prime}\right), \mathrm{H}-3^{\prime}\left(5^{\prime}\right)$ & 116.9 & $7.14, \mathrm{~d}(8.7)$ & $\mathrm{H}-2^{\prime}\left(6^{\prime}\right), \mathrm{H}-3^{\prime}\left(5^{\prime}\right)$ \\
\hline $4^{\prime}$ & 157.1 & $9.46 \mathrm{OH}$ & $\mathrm{H}-2^{\prime}\left(6^{\prime}\right), \mathrm{H}-3^{\prime}\left(5^{\prime}\right)$ & 157.1 & 年 & $\begin{array}{c}\mathrm{H}-2^{\prime}\left(6^{\prime}\right), \mathrm{H}-3^{\prime}\left(5^{\prime}\right) \\
\text { Glc2-H-1"' }\end{array}$ \\
\hline \multicolumn{7}{|l|}{$\begin{array}{l}\text { Glucose } \\
\text { moiety } 1\end{array}$} \\
\hline $1^{\prime \prime}$ & 100.8 & $5.32, \mathrm{~d}(3.5)$ & & 99.9 & $5.46, \mathrm{~d}(3.5)$ & \\
\hline $2^{\prime \prime}$ & 72.1 & $3.37, \mathrm{~m}$ & H-1", H-3", H-4" & 72.1 & $3.39, \mathrm{~m}$ & H-1", H-3" \\
\hline 3" & 73.2 & $3.73, \mathrm{~m}$ & H-1", H-2", H-4" & 73.3 & $3.74, \mathrm{~m}$ & H-1", H-2", H-4" \\
\hline $4^{\prime \prime}$ & 70.1 & $3.23, \mathrm{~m}$ & H-3", H-5", H-6" & 70.0 & $3.21, \mathrm{~m}$ & H-3", H-5", H-6" \\
\hline $5^{\prime \prime}$ & 72.2 & $3.77, \mathrm{~m}$ & H-1", H-3", H-6" & 74.2 & $3.48, \mathrm{~m}$ & H-1", H-6" \\
\hline $6 "$ & 66.1 & $\begin{array}{l}3.73, \mathrm{~m} \\
3.59, \mathrm{~m}\end{array}$ & $\begin{array}{l}\text { H-4", H-5", } \\
\text { Glc2-H-1"' }\end{array}$ & 60.9 & $\begin{array}{l}3.56, \mathrm{~m} \\
3.48, \mathrm{~m}\end{array}$ & H-4", H-5" \\
\hline \multicolumn{7}{|l|}{$\begin{array}{l}\text { Glucose } \\
\text { moiety } 2\end{array}$} \\
\hline $1^{\prime \prime \prime}$ & 98.2 & $4.65, \mathrm{~d}(3.5)$ & Glc1-H-6" & 98.1 & $5.42, \mathrm{~d}(3.5)$ & \\
\hline $2^{\prime \prime \prime}$ & 71.9 & $3.18, \mathrm{~m}$ & H-1"', H-3"' & 71.8 & $3.39, \mathrm{~m}$ & H-1"', H-3"' \\
\hline $3^{\prime \prime \prime}$ & 73.2 & $3.43, \mathrm{~m}$ & $\mathrm{H}-1^{\prime \prime \prime}, \mathrm{H}-2^{\prime \prime \prime}, \mathrm{H}-4^{\prime \prime \prime}$ & 73.3 & $3.64, \mathrm{~m}$ & $\mathrm{H}-1^{\prime \prime \prime}, \mathrm{H}-2^{\prime \prime \prime}, \mathrm{H}-4^{\prime \prime \prime}$ \\
\hline $4^{\prime \prime \prime}$ & 70.1 & $3.11, \mathrm{~m}$ & H-3"', H-5"', H-6"' & 70.1 & $3.21, \mathrm{~m}$ & $\mathrm{H}-3^{\prime \prime \prime}, \mathrm{H}-5^{\prime \prime \prime}, \mathrm{H}-6^{\prime \prime \prime}$ \\
\hline $5^{\prime \prime \prime}$ & 72.4 & $3.49, \mathrm{~m}$ & H-1"', H-3"', H-6"' & 73.9 & $3.48, \mathrm{~m}$ & H-1"' \\
\hline $6^{\prime \prime \prime}$ & 60.8 & $\begin{array}{l}3.60, \mathrm{~m} \\
3.48, \mathrm{~m}\end{array}$ & H-4"', H-5"' & 60.8 & $\begin{array}{l}3.60, \mathrm{~m} \\
3.48, \mathrm{~m}\end{array}$ & $\mathrm{H}-4^{\prime \prime \prime}, \mathrm{H}-5^{\prime \prime \prime}$ \\
\hline
\end{tabular}

\subsection{Characterization of the 8-OHDe Diglucosides}

Glycosylation could improve the aqueous solubility and stability of 8-OHDe, and hence its clinical application. Herein, the aqueous solubility of 8-OHDe and its diglucoside derivatives, 8-OHDe-7-G2 (1) and 8-OHDe-7-G-4'-G (2), was determined using previously described methods [13]. The intermediate 8-OHDe-7-G2 (1) yield was low; thus, its aqueous solubility could not be accurately determined. Nevertheless, Table 2 shows that the aqueous solubility of 8-OHDe-7-G2 (1) was higher than the detectable maximum $(10,000 \mathrm{mg} / \mathrm{L})$, which was at least $704-$ fold higher than that of 8 -OHDe $(14.2 \mathrm{mg} / \mathrm{L})$. The aqueous solubility of 8-OHDe-7-G-4'-G (2) $(65,598 \mathrm{mg} / \mathrm{L})$ was 4619 -fold higher than that of 8-OHDe. The solubility of the two 8-OHDe diglucosides was much higher than that of 8-OHDe-7-G, a monoglucoside [13]. In addition, in this study, the aqueous solubility of 8-OHDe-7-G-4'-G (2) was 7-fold higher than that of DA2 (8907 mg/L) reported by Rha et al., (2019) [16]. Such water solubility differences between the two isoflavone diglucosides may be caused by the 
two distant glucosyl sites (C7 and $\left.\mathrm{C} 4^{\prime}\right)$ of 8-OHDe-7-G-4'-G (2). The mono-sugar linked to the two glucosyl sites (C7 and C4') of 8-OHDe-7-G-4'-G (2) may become more soluble than the di-sugar linked to the one glucosyl site (C7) of DA2. Previous reports revealed that the higher water solubility of isoflavone glucoside derivatives can improve the oral bioavailability of the original molecules $[10,19,20]$. Therefore, of all the derivatives, ultrahighly soluble 8-OHDe-7-G-4'-G (2) has the greatest potential for future pharmaceutical and cosmeceutical applications.<smiles>O=c1c(-c2ccc(O)cc2)coc2c(O)c(O)ccc12</smiles>

8-OHDe

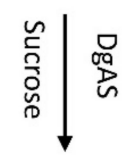<smiles>O=c1c(-c2ccc(O)cc2)coc2c(O)c(OC3OC(CO)C(O)C(O)C3O)ccc12</smiles>

8-OHDe-7-O- $\alpha$-glucopyranoside (8-OHDe-7-G)

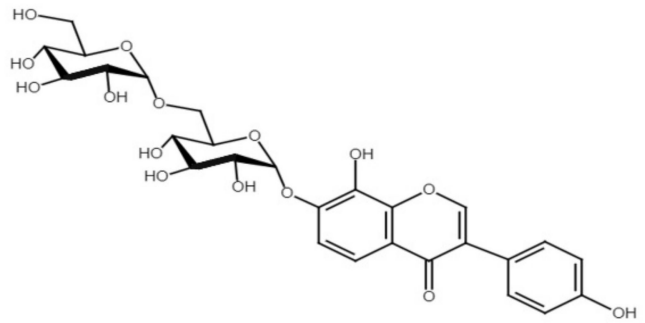

8-OHDe-7-O-[ $\alpha$-glucopyranosyl-( $1 \rightarrow 6)$ - $\alpha$-glucopyranoside] (1) (8-OHDe-7-G2)
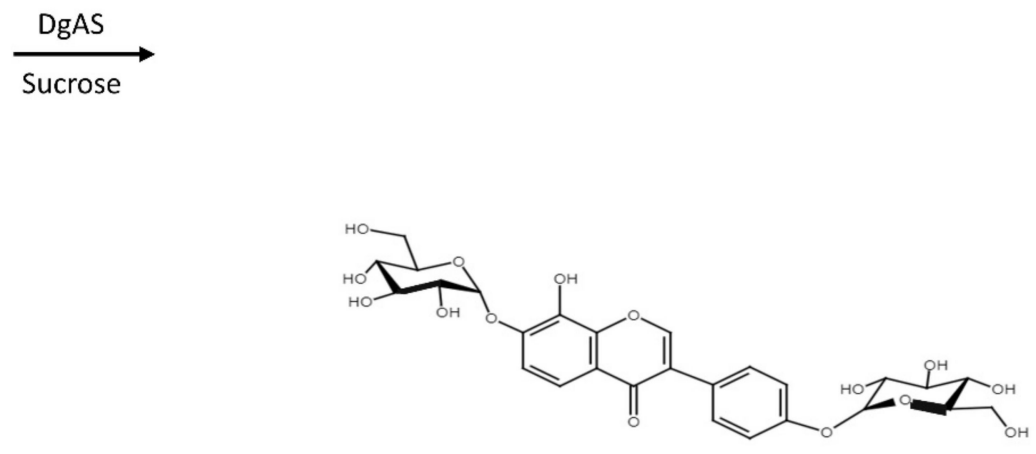

8-OHDe-7, 4'-O- $\alpha$-diglucopyranoside (2) (8-OHDe-7-G-4'-G)

Figure 3. The biotransformation of 8-OHDe mediated by DgAS.

Table 2. Characterization of 8-OHDe and its glucoside derivatives.

\begin{tabular}{ccc}
\hline Compound & Aqueous Solubility (mg/L) & Stability in Alkaline Solution $^{2}$ \\
\hline 8-OHDe & $14.2 \pm 5.4\left(1\right.$-fold) ${ }^{1}$ & $10.9 \pm 3.7$ \\
8-OHDe-7-G & $125.4 \pm 3.6\left(8.8\right.$-fold) ${ }^{1}$ & $94.6 \pm 3.2^{3}$ \\
8-OHDe-7-G2 (1) & $>10,000\left(>704.2\right.$-fold) ${ }^{1}$ & $94.4 \pm 3.6$ \\
8-OHDe-7-G-4'-G (2) & $65,598.4\left(4619.6\right.$-fold) ${ }^{1}$ & $92.8 \pm 5.0$ \\
\hline
\end{tabular}

${ }^{1}$ The fold increase in aqueous solubility of the 8 -OHDe glucoside derivatives is expressed as relative to that of 8-OHDe, normalized to $1 .{ }^{2}$ Stability is presented as the $\%$ of residual compound after $96 \mathrm{~h}$ in $50 \mathrm{mM}$ Tris buffer (pH 8). ${ }^{3}$ Data obtained from Chang et al. [13].

In addition to its low solubility, 8-OHDe is unstable in alkaline solutions-a property that limits its application [9]. Thus, the stability of the two 8-OHDe diglucosides was also studied. Table 2 shows that over $92 \%$ of the 8 -OHDe diglucosides remained in $50 \mathrm{mM}$ 
Tris buffer ( $\mathrm{pH} 8.0$ ) after a $96 \mathrm{~h}$ incubation at $25^{\circ} \mathrm{C}$. By contrast, only $10 \%$ of 8 -OHDe remained under the same conditions. The stabilities of the two 8-OHDe diglucosides were equal to that of the 8-OHDe monoglucoside (8-OHDe-7-G) [13]. The results indicate that 8-OHDe glucosides are stable in alkaline solutions because of the modification of the 7,8-ortho-diphenol structure of 8-OHDe. Some studies have indicated that ortho-hydroxyl substitutions, whether on the B- or A-ring, are the most important feature influencing the antioxidant activity of flavonoid compounds [21,22]. Therefore, the high antioxidant activity of 8-OHDe may be due to the 7,8-ortho-dihydroxyl groups in the A-ring [1], although this conformation would lead to 8-OHDe being unstable. Contrastingly, in the 8-OHDe glucosides, the ortho-hydroxyl groups were glycosylated, and the ortho-dihydroxyl groups disappeared. For this reason, both 8-OHDe diglucosides were found to be considerably more stable than 8-OHDe in solution. In short, the glycosylation of 8-OHDe increases its stability in alkaline conditions, and the two novel 8-OHDe diglucosides could be utilized in future bioindustrial activities.

A recent study indicated that in vitro enzymatically $O$-glycosylated flavonoids could be deglycosylated and reverted to their parent (pre-glycosylation) compounds under intestinal conditions [23]. In other studies, in vitro enzymatically $O$-glycosylated flavonoids reverted to their parent molecules under in vitro [23] or in vivo [24] fecal fermentation conditions, in which a variety of bacterial species metabolize sugars of flavonoid glycosides, such as glucose, that serve as easily accessible energy sources. On the other hand, in vitro enzymatically glycosylated flavonoids possess higher solubility and stability than their parent molecules. Thus, glycosylation is viewed as an attractive feature that could be exploited to enhance bioavailability and improve the cellular absorption of consumed flavonoids $[19,20]$. Therefore, the findings of these reports indicate that, upon oral ingestion, the two 8-OHDe glucosides identified in this study might also be digested to produce 8OHDe, which could then be absorbed and exert its wide range of pharmaceutical activities. Moreover, the two stable and highly water-soluble 8-OHDe glucosides might possess higher bioavailability than 8-OHDe, as found in previous studies.

\section{Conclusions}

Two novel soluble 8-OHDe diglucosides, 8-OHDe-7-G2 (1) and 8-OHDe-7-G-4'-G (2), were produced by the glycosylation of 8-OHDe by DgAS with an extended reaction time. The two 8-OHDe diglucosides were found to be highly stable in alkaline solutions. Based on the multiple bioactivities of 8-OHDe, the two new 8-OHDe diglucosides have the potential for pharmacological usage in the future.

Supplementary Materials: The following are available online at https:/ /www.mdpi.com/article/10 .3390/fermentation7040232/s1: Figure S1, Sodium dodecyl sulfate polyacrylamide gel electrophoresis (SDS-PAGE) analysis of purified DgAS; Figure S2, High-performance liquid chromatography (HPLC) analysis of the biotransformation products of 8-OHDe produced by heat-inactivated DgAS; Figure S3, Mass analysis of compound (1); Figure S4, Mass analysis of compound (2); Figure S5, Mass analysis of compound (3); Figure S6, The ${ }^{1} \mathrm{H}-\mathrm{NMR}$ (700 MHz, DMSO- $d_{6}$ ) spectrum of compound (1); Figure S7, The ${ }^{13} \mathrm{C}-\mathrm{NMR}\left(176 \mathrm{MHz}, \mathrm{DMSO}-d_{6}\right)$ spectrum of compound (1); Figure S8, The DEPT-135 (176 MHz, DMSO- $d_{6}$ ) spectrum of compound (1); Figure S9, The H-H COSY (700 MHz, DMSO- $d_{6}$ ) spectrum of compound (1); Figure S10, The H-H NOESY (700 MHz, DMSO- $d_{6}$ ) spectrum of compound (1); Figure S11, The HSQC (700 MHz, DMSO- $d_{6}$ ) spectrum of compound (1); Figure S12, The HMBC (700 MHz, DMSO- $d_{6}$ ) spectrum of compound (1); Figure S13, The ${ }^{1} \mathrm{H}-\mathrm{NMR}\left(700 \mathrm{MHz}, \mathrm{DMSO}-d_{6}\right)$ spectrum of compound (2); Figure S14, The ${ }^{13} \mathrm{C}-\mathrm{NMR}\left(176 \mathrm{MHz}, \mathrm{DMSO}-d_{6}\right)$ spectrum of compound (2); Figure S15, The DEPT-135 (176 MHz, DMSO- $d_{6}$ ) spectrum of compound (2); Figure S16, The H-H COSY (700 MHz, DMSO- $d_{6}$ ) spectrum of compound (2); Figure S17, The H-H NOESY (700 MHz, DMSO- $d_{6}$ ) spectrum of compound (2); Figure S18, The HSQC (700 MHz, DMSO- $d_{6}$ ) spectrum of compound (2); Figure S19, The HMBC (700 MHz, DMSO- $d_{6}$ ) spectrum of compound (2); and Figure S20, The key HMBC and NOESY correlations of compounds (1) and (2).

Author Contributions: Conceptualization, T.-S.C.; data curation and methodology, S.-Y.L., Y.-R.Z., T.-S.C. and C.-M.C.; project administration, T.-S.C. and J.-Y.W.; writing of original draft, T.-S.C., 
T.-Y.W., J.-Y.W. and C.-M.C.; writing of review and editing, T.-S.C., T.-Y.W., J.-Y.W. and C.-M.C. All authors have read and agreed to the published version of the manuscript.

Funding: This study was supported by grants from the Ministry of Science and Technology in Taiwan (project no. MOST 110-2221-E-024-002 to T.-S.C.; MOST 110-2221-E-507-002 to J.-Y.W. MOST 110-2637-E-041-002 to C.-M.C.).

Institutional Review Board Statement: Not applicable.

Informed Consent Statement: Not applicable.

Data Availability Statement: The data presented in this study are available in the article or Supplementary Materials.

Conflicts of Interest: The authors declare no conflict of interest.

\section{References}

1. Chang, T.S. Isolation, bioactivity, and production of ortho-hydroxydaidzein and ortho-hydroxygenistein. Int. J. Mol. Sci. 2014, 15, 5699-5716. [CrossRef] [PubMed]

2. Wu, P.S.; Ding, H.Y.; Yen, J.H.; Chen, S.F.; Lee, K.H.; Wu, M.J. Anti-inflammatory activity of 8-hydroxydaidzein in LPS-stimulated BV2 microglial cells via activation of Nrf2-antioxidant and attenuation of Akt/NF-kappaB-inflammatory signaling pathways, as well as inhibition of COX-2 activity. J. Agric. Food Chem. 2018, 66, 5790-5801. [CrossRef]

3. Kim, E.; Kang, Y.G.; Kim, J.H.; Kim, Y.J.; Lee, T.R.; Lee, J.; Kim, D.; Cho, J.Y. The antioxidant and anti-inflammatory activities of 8-hydroxydaidzein (8-HD) in activated macrophage-like RAW264.7 cells. Int. J. Mol. Sci. 2018, 19, 1828. [CrossRef]

4. $\quad$ Lim, T.G.; Kim, Y.A.; Kim, J.E.; Baek, S.; Lee, S.Y.; Lee, C.C.; Chen, H.; Kim, J.R.; Kwon, J.Y.; Bode, A.M.; et al. PKCiota is a target of 7,8,4'-trihydroxyisoflavone for the suppression of UVB-induced MMP-1 expression. Exp. Dermatol. 2018, 27, 449-452. [CrossRef]

5. Ko, Y.H.; Kim, S.K.; Kwon, S.H.; Seo, J.Y.; Lee, B.R.; Kim, Y.J.; Hur, K.H.; Kim, S.Y.; Lee, S.Y.; Jang, C.G. 7,8,4'-trihydroxyisoflavone, a metabolized product of daidzein, attenuates 6-hydroxydopamine-induced neurotoxicity in SH-SY5Y cells. Biomol. Ther. 2019, 27, 363-372. [CrossRef]

6. Ko, Y.H.; Kwon, S.H.; Ma, S.X.; Seo, J.Y.; Lee, B.R.; Kim, K.; Kim, S.Y.; Lee, S.Y.; Jang, C.G. The memory-enhancing effects of 7,8,4'-trihydroxyisoflavone, a major metabolite of daidzein, are associated with activation of the cholinergic system and BDNF signaling pathway in mice. Brain Res. Bull. 2018, 142, 197-206. [CrossRef]

7. Wu, S.C.; Chang, C.W.; Lin, C.W.; Hsu, Y.C. Production of 8-hydroxydaidzein polyphenol using biotransformation by Aspergillus oryzae. Food Sci. Technol. Res. 2015, 21, 557-562. [CrossRef]

8. Seo, M.H.; Kim, B.N.; Kim, K.R.; Lee, K.W.; Lee, C.H.; Oh, D.K. Production of 8-hydroxydaidzein from soybean extract by Aspergillus oryzae KACC 40247. Biosci. Biotechnol. Biochem. 2013, 77, 1245-1250. [CrossRef]

9. Chang, T.S. 8-Hydroxydaidzein is unstable in alkaline solutions. J. Cosmet. Sci. 2009, 60, 353-357. [CrossRef] [PubMed]

10. Zhao, J.; Yang, J.; Xie, Y. Improvement strategies for the oral bioavailability of poorly water-soluble flavonoids: An overview. Int. J. Pharm. 2019, 570, 118642. [CrossRef] [PubMed]

11. Hofer, B. Recent developments in the enzymatic O-glycosylation of flavonoids. Appl. Microbiol. Biotechnol. 2016, 100, 4269-4281. [CrossRef]

12. Chiang, C.M.; Wang, T.Y.; Yang, S.Y.; Wu, J.Y.; Chang, T.S. Production of new isoflavone glucosides from glycosylation of 8-hydroxydaidzein by glycosyltransferase from Bacillus subtilis ATCC 6633. Catalysts 2018, 8, 387. [CrossRef]

13. Chang, T.S.; Wang, T.Y.; Yang, S.Y.; Kao, Y.H.; Wu, J.Y.; Chiang, C.M. Potential industrial production of a well-soluble, alkalinestable, and anti-inflammatory isoflavone glucoside from 8-hydroxydaidzein glucosylated by recombinant amylosucrase of Deinococcus geothermalis. Molecules 2019, 24, 2236. [CrossRef]

14. Moulis, C.; Guieysse, D.; Morel, S.; Severac, E.; Remaud-Simeon, M. Natural and engineered transglycosylases: Green tools for the enzyme-based synthesis of glycoproducts. Curr. Opin. Chem. Biol. 2021, 61, 96-106. [CrossRef] [PubMed]

15. Seo, D.H.; Yoo, S.H.; Choi, S.J.; Kim, Y.R.; Park, C.S. Versatile biotechnological applications of amylosucrase, a novel glucosyltransferase. Food Sci. Biotechnol. 2020, 29, 1-16. [CrossRef]

16. Rha, C.S.; Kim, E.R.; Kim, Y.J.; Jung, Y.S.; Kim, D.O.; Park, C.S. Simple and efficient production of highly soluble daidzin glycosides by amylosucrase from Deinococcus geothermalis. J. Agric. Food Chem. 2019, 67, 12824-12832. [CrossRef]

17. Rha, C.S.; Kim, H.G.; Baek, N.I.; Kim, D.O.; Park, C.S. Using amylosucrase for the controlled synthesis of novel isoquercitrin glycosides with different glycosidic linkages. J. Agric. Food Chem. 2020, 68, 13798-13805. [CrossRef]

18. Tian, Y.Q.; Xu, W.; Zhang, W.L.; Zhang, T.; Guang, C.E.; Mu, W.M. Amylosucrase as a transglucosylation tool: From molecular features to bioengineering applications. Biotechnol. Adv. 2018, 36, 1540-1552. [CrossRef]

19. Huang, G.; Lv, M.; Hu, J.; Huang, K.; Xu, H. Glycosylation and activities of natural products. Mini-Rev. Med. Chem. 2016, 16, 1013-1016. [CrossRef]

20. Fu, J.; Wu, Z.; Zhang, L. Clinical applications of the naturally occurring or synthetic glycosylated low molecular weight drugs. Prog. Mol. Biol. Transl. Sci. 2019, 163, 487-522. [CrossRef] [PubMed] 
21. Sordon, S.; Poplonski, J.; Milczarek, M.; Stachowicz, M.; Tronina, T.; Kucharska, A.Z.; Wietrzyk, J.; Huszcza, E. Structureantioxidant-antiproliferative activity relationships of natural C7 and C7-C8 hydroxylated flavones and flavanones. Antioxidants 2019, 8, 210. [CrossRef] [PubMed]

22. Treml, J.; Smejkal, K. Flavonoids as potent scavengers of hydroxyl radicals. Compr. Rev. Food Sci. Food Saf. 2016, 15, 720-738. [CrossRef] [PubMed]

23. Jang, D.; Jung, Y.S.; Seong, H.; Kim, M.S.; Rha, C.S.; Nam, T.G.; Han, N.S.; Kim, D.O. Stability of enzyme-modified flavonoid Cand O-glycosides from common buckwheat sprout extracts during in vitro digestion and colonic fermentation. J. Agric. Food Chem. 2021, 69, 5764-5773. [CrossRef] [PubMed]

24. Hein, E.M.; Rose, K.; Slot, G.V.; Friedrich, A.W.; Humpf, H.U. Deconjugation and degradation of flavonol glycosides by pig cecal microbiota characterized by fluorescence in situ hybridization (FISH). J. Agric. Food Chem. 2008, 56, 22812290. [CrossRef] [PubMed] 